c. Staphylococcus aureus, especially in cases associated with phlyctenules around or on the cornea.

d. Streptococcus.-Mercurochrome I per cent or 2 per cent is useful. Suphonamides can be applied locally and generally.

e. Gonococcus.-Gives rise to an extremely acute purulent conjunctivitis with very swollen lids, oedema of the conjunctiva, and much yellowish pus. It is extremely contagious, and often gives rise to a severe keratitis which may end in perforation and destruction of the eye.

Great trouble must be taken to prevent spread to the other eye, by covering the affected one with a Buller's shield.

The eye should be washed out four hourly with normal saline, and the lids painted with silver nitrate 2 per cent, providing this can be done without undue risk of injury to the cornea. In cases where the lids are so swollen that the eye is difficult to get at, and folds of conjunctiva are becoming strangled, it may be necessary to divide the outer canthus to give more room (canthotomy). Atropine should be applied if there is the slightest sign of corneal involvement. In addition to these older methods, the use of sulphonamides internally and externally is of great value.

The causative gonorrhoea must of course be energetically treated.

Ophthalmia neonatorum is often due to the gonococcous, though sometimes a condition just as severe can be caused by other organisms, especially the streptococcus. Treatment as for adults, except that a Buller's shield is not used.

\title{
THE PLACE OF ORTHOPTICS IN MODERN OPHTHALMOLOGICAL PRACTICE
}

\author{
By MARY B. DAVIES, B.A.(Lond.) \\ (Member of the British Orthoptic Society)
}

For many years now, particularly during the last century, attempts have been made to obtain a functional cure in cases of strabismus. More often than not, pioneers in orthoptics have met with failure for two reasons: (I) not enough was known about the aetiology of the condition; (2) the only apparatus available demanded altogether too much concentration on the part of the patient. In consequence of this, orthoptic practice as a whole fell into disrepute and it was not until several years after the appearance of a really good instrument that the first hospital department was opened. Nevertheless many schools of thought decried this modern treatment on the supposition that it claimed to cure all and every case of squint. This is grossly untrue, and has caused much misunderstanding.

There appears to be a prevalent idea that orthoptics dispense entirely with operation. Certainly there are some instances where a complete cure is effected by exercises alone, whereas formerly surgery would have been the only resort. In the main, however, the crux of the matter is this: there are roughly three types of case. Some respond to orthoptic treatment only, others require surgical help as well, while there still remain those for whom a cosmetically good result, either with or without operation, is the only hope.

The' chief differences between earlier practice and that in general operation to-day lie $(a)$ in the treatment meted out to cases in the first two categories, and $(b)$ in the more specialised selection of the cases. Improved technique is gradually perfecting the treatment suitable for selected cases, and increased knowledge of the subject is making this selection easier.

The question now arises as to who shall decide which case shall be treated and which refused. Obviously the responsibility must rest with the ophthalmic surgeon in charge of any given case, but he is generally too busy to carry out the necessarily minute tests. It is, therefore, generally desirable that as many cases as possible of muscle imbalance should be referred to the orthoptist. Her training includes a certain knowledge of elementary anatomy, physiology, and optics, besides practical orthoptics. She should, therefore, be able to make adequate tests, diagnose the condition in detail, and, after some experience, decide what chance there is of developing binocular vision. The aim of orthoptic treatment is to develop perfect binocular vision, and this possibility must therefore be explored at the outset. 
In the selection of cases a number of factors must be taken into account, especially the history and cause of the condition in each individual. For example, a congenital defect is less likely to develop into full binocular vision than one which has occurred later in life, when the binocular faculty only needs re-educating. Thus, a child who gave a birth history would be given a trial of the treatment, while an adult with a similar history would not, because if the former did not develop true fusion he would easily suppress one image, and no harm would be done, whereas an adult might be left with permanent diplopia. In the same way a child with amblyopia ex anopsia* would be "occluded," an adult would not. With regard to operation, a patient of any age who only squinted occasionally, i.e. on accommodation, or for some other reason, would normally respond to orthoptic treatment alone. Where a residual angle was always present, operation would almost certainly have to be undertaken, either in conjunction with treatment or not, according to the history.

This is no place to go into details of treatment, but mention may be made of some salient points. In the first place, "occlusion" is desirable in all cases of amblyopia ex anopsia* occurring in children up to the age of eight. Where the vision in one eye is less than $6 / 18$ on the Snellen test type the good eye should be totally occluded. Some variety of patent occluder may be used, but most children tend to peep round these, and it is better to stick a patch of plaster, either adhesive tape, elastoplast or isinglass, on to the face: provided the patch is changed frequently and the child is carefully supervised, no harm ensues from this practice. Thereafter, some form of partial occlusion, either by atropin or by patches on the spectacles, must follow until the vision is equal in both eyes. Actually atropin is especially valuable in cases of very young children, even before twelve months, for if it be inserted into the fixing eye as soon as the squint is noticed, it may obviate more drastic patching later on. Then again, atropin or partial occlusion may do much to promote full binocular vision by hampering the fixing eye and stimulating the one which is suppressed. Finally, various forms of paper occluders stuck on to the spectacles after operation are invaluable in helping to overcome slight residual angles. result.

It may be noted that here the skill of the orthoptist may be vital, even for a purely cosmetic

Special reference must now be made to the phenomenon of false projection, otherwise called false associated fixation, or abnormal retinal correspondence. It is particularly prevalent among tonic types of convergent squint. It involves the macula of the fixing eye and a noncorresponding part of the retina of the other eye. It exists whichever eye fixes, and is therefore a binocular condition. False projection can only be diagnosed by means of a major amblyoscope, which is also the instrument of choice in the treatment of this condition. Very careful stimulation of the macular area is required to break down the false projection, and it may be necessary to continue for twenty-four treatments. If at the end of that time the false projection still persists, an operation is indicated. It may so happen, after an operation, that the effect of the stimuli falling on each macula at the same time will automatically induce true projection, and that, if treatment is then continued, perfect binocular vision may result. This, however, is rare. The fact that it does occasionally occur should never be made an excuse for operating without previous treatment, because it is very difficult to work on the false projection without increasing the angle again.

Experience seems to indicate that all adults with a squint of long standing, children with an angle of more than fifty degrees, or with excessive hyperphoria, congenital alternators, of whateyer age, and cases of persistent amblyopia should be referred back to the surgeon without treatment. Only a cosmetic result is anticipated in most of these cases, but they should all be tested by an orthoptist afterwards so that no opportunity of developing binocular vision may be lost.

All other cases of muscle imbalance should be given a trial of treatment. Six visits are usually sufficient to indicate to what extent orthoptics will be beneficial. Where the angle is large or tonic, the aim of the orthoptist will be to develop as much binocular vision at the angle of the squint as possible, and some duction power. She will advise the surgeon when this has been achieved. With his aid, followed by further treatment, she will expect a good binocular result. Formerly a cosmetic effect only would have been looked for, and then, in the absence of binocular stimulation to maintain the parallel position, there was always a fear that the deviation might recur.

* Amblyopia ex anopsia denotes " a condition of blunting of vision or impairment of visual acuity due to disuse of one eye."-Lyle and Jackson. 
There is, however, a much larger number of cases which can be cured by orthoptics alone. These include accommodative types of convergent strabismus, the majority of divergent squints and nearly all forms of heterophoria or latent muscle imbalance. In all these instances the patient's eyes are quite straight at times. It would take too long to explain why the deviation takes place, but some indication of the general lines of treatment may be given. To cure an accommodative type will take anything up to fifty treatments. These cases generally "suppress" one eye or the other when they exert the power of accommodation unless they are allowed to converge at the same time. The aim of treatment is, therefore, to dissociate the two functions in the patient's mind. Bi-weekly attendances for treatment on a major amblyoscope, coupled with homework exercises, compatible with the patient's age and mentality, are indicated. Treatment should be begun at the earliest possible age, but it cannot be completed until a capacity to read easily is reached, so that all concentration may'be centred on the eyes. Consequently this type of squint may have to be tackled at intervals over a number of years. This may sound very tedious, but a spectacular improvement, which can usually be demonstrated quite early, helps considerably in rousing the enthusiasm of both parent and child. This type responds more readily than any other to orthoptic skill because it is entirely functional in character. When the time comes for discharge the patient should be binocular both for near and distant vision; he should possess a binocular visual acuity with glasses equal to the vision in each eye separately. Without glasses he should have a binocular visual acuity approximately one line less than with each eye separately. It follows that he should be able to leave off his glasses for most purposes except close work.

In the treatment of divergent squint the main object is to overcome "suppression" at the angle of deviation, so that diplopia will be experienced whenever squinting takes place. These patients usually develop good fusion and stereoscopic vision: they can then be taught adduction to 40 degrees at least. The angle tends to remain in many cases, but orthophoria can be maintained for most tests, and symptoms seldom arise after treatment.

Heterophoria has now been classified into at least five types:

I. Exophoria, or latent deviation of the visual axes outwards.

2. Esophoria, or latent deviation inwards.

3. Hyperphoria, or latent vertical deviation.

4. Cyclophoria, or deviation round a sagittal axis.

5. Convergence insufficiency, which explains itself.

In all these cases binocular vision is only maintained under great stress, and the excessive nervous energy involved often produces great discomfort. Formerly lenses, usually embodying prisms, would have been ordered. Latterly a course of orthoptic treatment has been found to effect a more lasting cure. From six to twenty-four treatments are usually sufficient. The aim is to overcome suppression, strengthen fusion, and generally tone up the muscles. A factor of additional importance is the psychological effect on these patients, who are not infrequently of a neurotic type. If regular personal attendance is inconvenient, homework exercises, under occasional supervision, are almost equally efficacious, provided the patient has a suitably persevering nature.

Finally, there must be taken into account those people who aspire to service in the R.A.F., etc., but who are debarred on account of a very small imbalance which does not even produce slight symptoms. A short course of orthoptic training will usually enable them to pass the most stringent occular tests.

Orthoptics now hold a valuable and quite well-defined position in modern ophthalmic practice. It must be emphasised again that cases must be carefully selected. Probably the most satisfactory procedure is for all cases of muscle imbalance to be referred to the orthoptic department unless the surgeon has previously decided that for some reason such as displaced lens, nystagmus, pronounced general disease, etc., they are totally unsuitable. In these days, when the wearing of spectacles is such a handicap in the commercial world, every effort should be made to dispense with them wherever possible. Also, at a time when unorthodox practices for abolishing lenses are so much in vogue, it seems advisable that a body of highly-trained and skilled technicians, under the aegis of the medical profession, should be given every opportunity of putting their art into practice. 